Check for updates

Cite this: RSC Adv., 2021, 11, 7416

DOI: $10.1039 / \mathrm{d} 1 \mathrm{ra90081a}$

rsc.li/rsc-advances

\section{Correction: Investigation of plasma metabolomics and neurotransmitter dysfunction in the process of Alzheimer's disease rat induced by amyloid beta 25- 35}

\author{
Mengying Wei, ${ }^{a b}$ Yuanyuan Liu, ${ }^{a}$ Zifeng Pi, ${ }^{b}$ Kexin Yue, ${ }^{a}$ Shizhe $\mathrm{Li}^{\mathrm{c}}$ Mingxin $\mathrm{Hu}{ }^{\mathrm{a}}$ \\ Zhiqiang Liu, ${ }^{\mathrm{b}}$ Fengrui Song ${ }^{\mathrm{b}}$ and Zhongying Liu*a
}

Correction for 'Investigation of plasma metabolomics and neurotransmitter dysfunction in the process of Alzheimer's disease rat induced by amyloid beta 25-35' by Mengying Wei et al., RSC Adv., 2019, 9 , 18308-18319. DOI: 10.1039/C9RA00302A.

The authors regret that, due to personal negligence, Fig. 1d-NG-4 in this article was repeatedly uploaded as Fig. 1d-NG-2. The authors apologize to readers for this inaccuracy and the corrected figure is shown below. This correction does not affect the results, discussion or conclusion of the article.
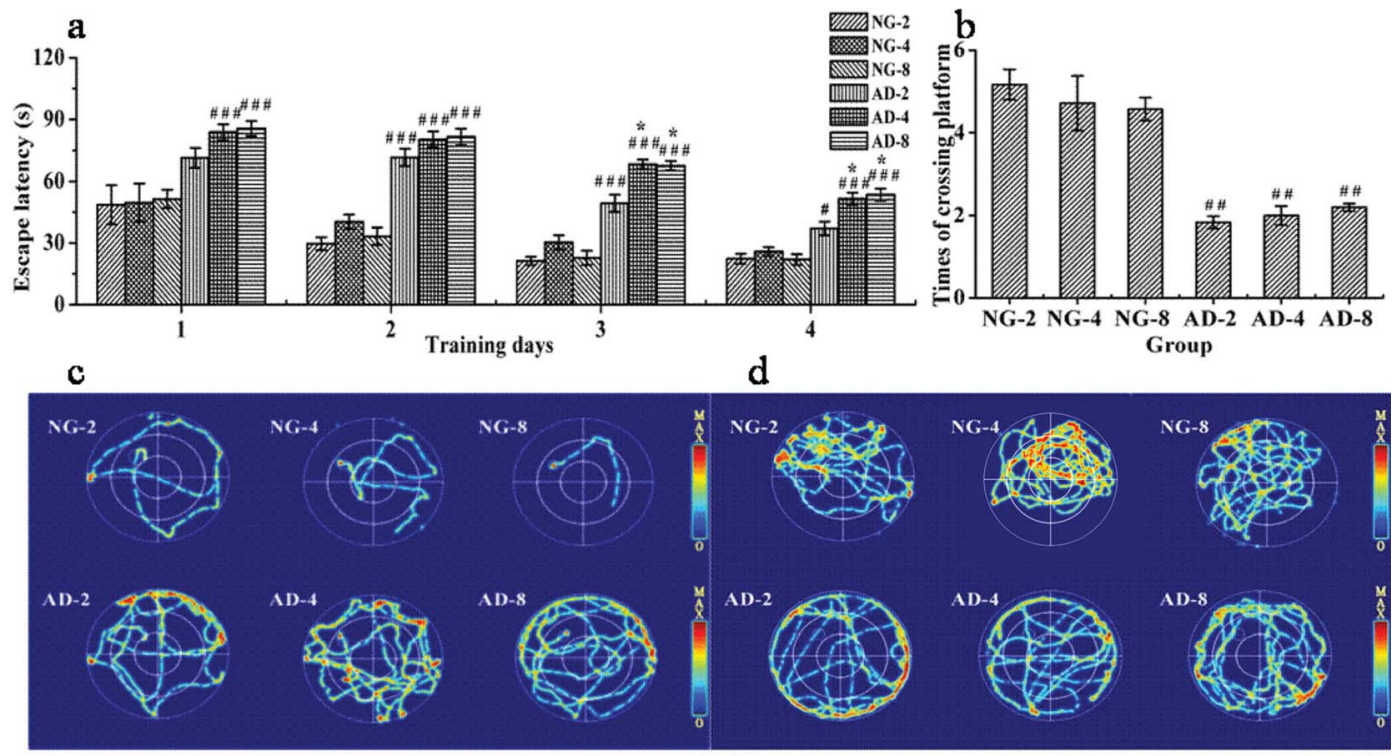

Fig. 1 The performance of spatial learning and memory in rats after 2, 4 and 8 weeks of modelling in the MWM test: (a) the escape latency during the 4 day training period, (b) times of crossing the original platform in the $120 \mathrm{~s}$ probe test, (c) trajectories of the last trial, (d) trajectories of rats from each group in (b). Notes: $n=10$, per group; data are expressed as mean \pm SEM, compared to NG by a $t$-test after the same week, \#\#\# $P<$ 0.001, ${ }^{\# \#} P<0.01,{ }^{\#} P<0.05$, compared to AD-2, ***P $<0.001, * * P<0.01, * P<0.05$, compared to AD-4, \&\&\& $P<0.001$, \&\& $P<0.01,{ }^{8} P<0.05$.

The Royal Society of Chemistry apologises for these errors and any consequent inconvenience to authors and readers.

${ }^{a}$ School of Pharmaceutical Sciences, Jilin University, 1266 Fujin Road, Changchun, 130021, China. E-mail: liuzy@jlu.edu.cn; Tel: +86 431 85619704

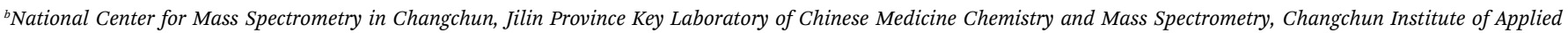
Chemistry, Chinese Academy of Sciences, Changchun 130022, China

'Guangdong Univ Technol, Inst Biomed \& Pharmaceut Sci, Guangzhou 510006, Guangdong, People's Republic of China 